\title{
Equações Diferenciais como Modelos Matemáticos de Dinâmicas Populacionais: um estudo voltado ao município de Santa Rosa/RS
}

\author{
Differential Equations as Mathematical Models of Population Dynamics: a study directed to \\ the municipality of Santa Rosa/RS
}

\begin{abstract}
Jonatan Ismael Eisermann
Universidade Federal de Santa Catarina (UFSC), Departamento de Matemática Programa de Pós-Graduação em Matemática Pura e Aplicada, Florianópolis, SC, Brasil http://orcid.org/0000-0001-8884-3480, jonatan.eisermann@hotmail.com
\end{abstract}

\author{
Gilberto Carlos Thomas \\ Instituto Federal de Educação, Ciência e Tecnologia Farroupilha (IFFAR) \\ Campus Santa Rosa, Santa Rosa, RS, Brasil \\ http://orcid.org/0000-0001-7492-6269, gilberto.thomas@iffarroupilha.edu.br
}

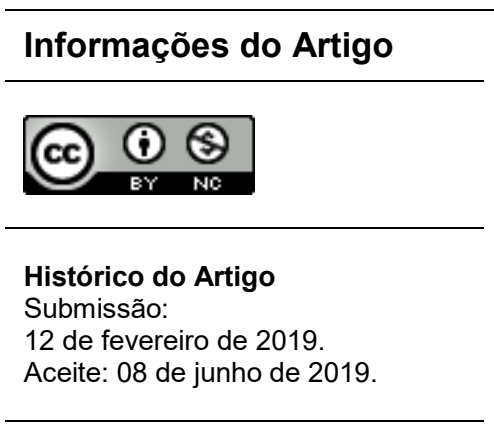

Palavras-chave

Equações Diferenciais

Dinâmica Populacional

Modelos Matemáticos

Santa Rosa

\section{Resumo}

O estudo sobre dinâmicas populacionais configura um dos principais instrumentos de planejamento e, consequentemente, de desenvolvimento político-econômico nas mais diversas esferas administrativas, principalmente devido à possibilidade de obter previsões e analisar interferências que possam otimizar determinado processo ao longo do tempo. Neste contexto, as Equações Diferenciais têm configurado um importante ramo de estudo na construção e no aprimoramento de modelos matemáticos que retratam dinâmicas populacionais. Visando sua exploração para prever a variação do número de habitantes do município de Santa Rosa/RS para as próximas cinco décadas, a presente pesquisa buscou analisar como tem ocorrido o comportamento desta relação no passado e identificar as variáveis que a interferem ou possam intervir no futuro, baseando-se na utilização dos modelos de Malthus, Verhulst e Gompertz. Trata-se, portanto, de uma pesquisa que alia a abordagem qualitativa à quantitativa, de cunho documental e bibliográfico, ancorada na exploração dos respectivos modelos matemáticos, mediante o reconhecimento histórico de sua construção e da conseguinte simulação de prováveis cenários populacionais santa-rosenses em um futuro próximo. Os resultados evidenciaram maior proximidade da variação populacional do município com o Modelo de Gompertz, induzindo a crer na existência de uma taxa de inibição da variável de estado proporcional ao respectivo logaritmo. Embora a taxa de crescimento da população santa-rosense tenha sido considerável em um passado recente, a previsão é que ela continue sendo positiva, porém em taxas menores, tendendo à estabilidade de um determinado limite sustentável.

\section{Abstract}

The study of population dynamics is one of the main instruments of planning and, consequently, of political and economic development in the most diverse administrative spheres, mainly due to the possibility of obtaining forecasts and analyzing interferences that can optimize a given process over time. In this context, Differential Equations have configured an important branch of study in the construction and improvement of mathematical models that portray population dynamics. Aiming at its exploration to predict the population variation of the municipality of Santa Rosa/RS for the next five decades, the present research sought to analyze how the behavior of this relationship has occurred in the past and identify the variables that interfere with it or may intervene in the future, based on the use of the Malthus, Verhulst and Gompertz models. It is, therefore, a research that combines the qualitative and quantitative approach, documental and bibliographical, anchored in the exploration of 
the respective mathematical models, through the historical recognition of its construction and the consequent simulation of probable population scenarios of Santa Rosa in a near future. The results showed a greater proximity of the population variation of the municipality with the Gompertz Model, inducing to believe in the existence of an inhibition rate of the state variable proportional to the respective logarithm. Although the growth rate of the Santa Rosa population has been considerable in the recent past, it is expected to remain positive, but at lower rates, tending to the stability of a certain sustainable limit.

\section{Introdução}

O histórico populacional do município de Santa Rosa, localizado na região noroeste do Rio Grande do Sul, evidencia a habitação primitiva por indígenas do grupo Tapes, o qual sofreu um sistema de redução para catequização por jesuítas e espanhóis a partir de 1626. Integrando o território dos Sete Povos das Missões, o município pertenceu, sucessivamente, a Porto Alegre, Rio Pardo e Santo Ângelo, tendo sua emancipação política no ano de 1931. Apesar disso, o processo de colonização só foi significativo a partir de 1915, quando entrou em execução um vasto plano de loteamento de terras para assentar uma maior quantidade de pessoas naquela região. Desde então, a população local tem buscado o desenvolvimento aliado ao seu crescimento populacional e econômico (SANTA ROSA, 2018).

Objetivando investigar como tem ocorrido a variação populacional do município em função do tempo, possibilitando a projeção de previsões demográficas futuras, buscou-se, com o presente estudo, utilizar dados históricos populacionais relativos aos últimos cinquenta anos para explorar alguns dos mais prestigiados modelos matemáticos que descrevem dinâmicas populacionais por meio de Equações Diferenciais: Malthus, Verhulst e Gompertz. Assim, foi possível verificar as principais potencialidades da utilização destes recursos matemáticos no processo de modelagem matemática, além dos próprios princípios e fundamentos que regem cada um dos modelos utilizados.

Desta forma, o presente trabalho é estruturado em três seções: na primeira, aborda-se o contexto de construção das equações supracitadas e suas respectivas soluções analíticas, a serem utilizadas na pesquisa; na segunda, discorre-se acerca da perspectiva metodológica utilizada, destacando as principais etapas e procedimentos adotados no estudo; na terceira, discute-se a construção de um modelo que se aproxime aos dados populacionais históricos de Santa Rosa, e sua conseguinte utilização na simulação de cenários populacionais futuros do município de Santa Rosa.

Convém salientar que a pesquisa faz parte de um projeto de iniciação científica, cadastrado e aprovado pelo Instituto Federal de Educação Ciência e Tecnologia Farroupilha, sob título de "Estudo e análise através de modelos matemáticos das tendências de crescimento populacional no município de Santa Rosa da Região Noroeste do Rio Grande do Sul", sendo desenvolvida pelos pesquisadores voluntários ao longo do segundo semestre de 2018. 


\section{Modelagem Matemática de Dinâmicas Populacionais}

A modelagem matemática pode ser entendida como a tentativa de representar, através de expressões matemáticas, fenômenos que o homem necessita compreender para poder interferir positivamente em seu processo. No Brasil, Rodney Carlos Bassanezi é um dos principais pesquisadores da área, e define-a como:

[...] um processo dinâmico utilizado para a obtenção e validação de modelos matemáticos. É uma forma de abstração e generalização com a finalidade de previsão de tendências. A modelagem consiste, essencialmente, na arte de transformar situações da realidade em problemas matemáticos cujas soluções devem ser interpretadas na linguagem usual (BASSANEZI, 2004, p. 24).

Quando se procura refletir sobre uma porção da realidade, na tentativa de explicar, de entender, ou de agir sobre ela, o processo usual é selecionar argumentos ou parâmetros considerados essenciais e formalizá-los por meio de um sistema artificial: o modelo (ibidem, p. 19). Conforme Beimbengut e Hein (2005), o modelo consiste na representação de um processo por meio da linguagem matemática, por isso envolve alguns procedimentos importantes na modelagem matemática: a) a interação, que necessita do reconhecimento do problema e a familiarização com o assunto que será modelado; b) a matematização, que implica na formulação das hipóteses a serem consideradas no problema; c) o modelo matemático, que pressupõe sua construção, posterior interpretação dos resultados e validação em relação aos objetivos traçados.

Assim, se o modelo não atender aos resultados esperados para sua validação, o processo de modelagem deve voltar a etapa de matematização, revendo as hipóteses e variáveis a fim de reformulá-lo até a sua efetiva validação. É importante lembrar que a modelagem matemática trabalha com aproximações da realidade, elaborando uma representação de um sistema ou parte dele. Por isso, o grau de aproximação da realidade é fator preponderante na validação do modelo e na busca em explicar padrões e funcionamentos dos mais diferentes processos que regem a vida no mundo.

Os primeiros registros de modelos matemáticos que descrevem dinâmicas populacionais se deram em meados do século XVIII, sendo, a partir disso, aprimorados e estudados por diversos pesquisadores da área. Neste contexto, ressalta-se a importância da utilização de Equações Diferenciais ${ }^{1}$, tendo em vista a exploração dos diferenciais, que permitem representar taxas de variação entre duas ou mais variáveis e, assim, projetar cenários futuros do fenômeno envolvido.

As projeções de população têm fundamental importância para o cálculo de indicadores sociodemográficos, servem de subsídios para a implementação de políticas públicas e a posterior avaliação de seus respectivos programas, bem como para estratégias de planejamento do setor privado. Além disso, possibilitam antecipar os desafios a serem enfrentados pela sociedade brasileira nas próximas décadas, assim como as oportunidades que surgirão em decorrência das mudanças demográficas (IBGE, 2018b, p. 7).

\footnotetext{
1 Uma Equação Diferencial é definida por uma relação matemática de igualdade, que contém as derivadas ou diferenciais de uma variável dependente, em relação a uma ou mais variáveis independentes.
} 
Atualmente, no Brasil, o Instituto Brasileiro de Geografia e Estatística (IBGE) é o responsável pelo recenseamento da população e pelo cálculo de projeções populacionais futuras, a nível nacional e estadual. Seus modelos baseiam-se nas evoluções e adaptações matemáticas em relação ao contexto de cada região considerada, servindo de meio para o planejamento estratégico para órgãos públicos e privados.

\subsection{Modelo de Malthus}

Ao descrever pela primeira vez a dinâmica populacional em linguagem matemática, Thomas Robert Malthus, em 1798, partiu do pressuposto de que a variação populacional $(d P)$ em relação ao tempo $(d t)$ é proporcional ao seu tamanho em cada instante $(P)$. Desta forma, assumiu-se que existia um coeficiente $k$, de crescimento $(k>0)$ ou decrescimento $(k<0)$, calculado a partir da diferença entre a taxa de natalidade e de mortalidade de determinada população, isto é:

$$
\frac{d P}{d t}=k P
$$

Resolvendo analiticamente (1) e designando $c$ uma constante, obteremos:

$$
\begin{aligned}
& \int \frac{d P}{P}=\int k d t \\
& \ln P=k t+c .
\end{aligned}
$$

O processo exponencial em (3), garante que:

$$
\begin{aligned}
& P=e^{k t+c}, \\
& P=e^{k t} e^{c} .
\end{aligned}
$$

Sendo $e^{c}$ uma constante de valor desconhecido, ela será denominada de $c_{1}$, levando encontrar a seguinte relação:

$$
P(t)=c_{1} e^{k t}
$$

Denominando $P_{0}$ a população no instante inicial $(t=0)$, tem-se que $P_{0}=c_{1}$, e, assim, a solução analítica do modelo de Malthus torna-se:

$$
P(t)=P_{0} e^{k t}
$$

Analisando a função, é fácil verificar que, dado a necessidade de $P_{0} \geq 0$, ter um coeficiente $k>0$ significa que a população crescerá e continuará expandindo para o infinito, isto é, 
$\lim _{t \rightarrow \infty} P(t)=+\infty$. Por outro lado, ter $k<0$, implica que a população tenderá a zero ou, em outras palavras, à extinção.

Por utilizar de uma função exponencial (7), Malthus defendia a ideia de que o crescimento da população humana ocorria em progressão geométrica, enquanto os meios de sobrevivência cresceriam em progressão aritmética (BASSANEZI, 2004). Assumia-se, assim, que seu modelo era incoerente a partir de determinado ponto, visto que no momento que os recursos não fossem mais suficientes para atender toda população, a tendência seria aumentar o número de mortes e, consequentemente, a função da população decresceria até estabilizar-se com a função linear que representa os meios de sobrevivência em relação ao tempo.

\subsection{Modelo de Verhulst}

O problema de infinidade do modelo malthusiano só foi resolvido em 1837, quando Pierre François Verhulst aprimorou-o acrescentando um fator limitante. O matemático supôs que a população deveria crescer até um limite máximo sustentável $L$, e, em seguida, tendia a se estabilizar. Algebricamente, ele representou:

$$
\frac{d P}{d t}=k P\left(1-\frac{P}{L}\right)
$$

De imediato, observa-se que quando $P$ é pequeno em relação à $L$, a equação reduz-se ao modelo exponencial de Malthus. Por outro lado, quando a população alcança o limite máximo $(P=$ $L$ ), não haverá taxa de crescimento nem decrescimento, isto é, a população se estabilizará em $L$.

Integrando ambos os lados da igualdade, a referida Equação Diferencial apresenta a seguinte solução analítica:

$$
\int \frac{d P}{P\left(1-\frac{P}{L}\right)}=\int k d t
$$

Como a expressão à esquerda da igualdade pode ser fracionada em partes, é possível considerar a seguinte situação:

$$
\int \frac{d P}{P\left(1-\frac{P}{L}\right)}=\int\left(\frac{1}{P}+\frac{\frac{1}{L}}{1-\frac{P}{L}}\right) d P
$$

E assim, dá-se continuidade à resolução de (9):

$$
\int\left(\frac{1}{P}+\frac{\frac{1}{L}}{1-\frac{P}{L}}\right) d P=\int k d t
$$


Nomeando $c$ como uma constante e explorando da propriedade logarítmica do quociente, obtêm-se:

$$
\begin{gathered}
\ln |P|-\ln \left|1-\frac{P}{L}\right|=k t+c, \\
\ln \left|\frac{P}{1-\frac{P}{L}}\right|=k t+c .
\end{gathered}
$$

Utilizando o processo exponencial em (13), considerando $e^{c}=c_{2}$ e usufruindo de manipulações algébricas, obtém-se:

$$
\begin{gathered}
\frac{P}{1-\frac{P}{L}}=e^{k t+c}, \\
\frac{P L}{L-P}=c_{2} e^{k t}, \\
P L+P c_{2} e^{k t}=L c_{2} e^{k t}, \\
P=\frac{L c_{2} e^{k t}}{L+c_{2} e^{k t} .}
\end{gathered}
$$

Ao considerar a condição inicial $P(0)=P_{0}$, verifica-se que:

$$
c_{2}=\frac{P_{0} L}{L-P_{0}}
$$

Assim, basta substituir (18) em (17) e encontrar:

$$
P(t)=\frac{L P_{0}}{\left(L-P_{0}\right) e^{-k t}+P_{0}}
$$

Ao analisar a função (19), percebe-se que, diferente do modelo de Malthus, $\lim _{t \rightarrow \infty} P(t)=L$.

Neste modelo, a taxa de variação da população tende a zero para grandes intervalos de tempo já que quando a população é menor do que a capacidade de suporte ela cresce e quando é maior que a capacidade de suporte ela diminui, ou seja, em ambos os casos a população tende a um valor de suporte representado no modelo pela constante $L$ (Adaptado de VILLAR, 2014, p. 19).

Apesar de superar o problema da tendência infinita, o modelo de Verhulst não é considerado ideal por não prever a possibilidade de extinção da espécie. 


\subsection{Modelo de Gompertz}

No mesmo século em que Verhulst desenvolveu sua equação, o matemático inglês Benjamin Gompertz propôs um modelo com princípios semelhantes, porém utilizando como limitador uma taxa de inibição da variável de estado proporcional ao respectivo logaritmo. Tal fato implica em uma taxa de crescimento maior no início do processo, alterando rápida e progressivamente para um crescimento mais lento. A Equação Diferencial tomada como base foi:

$$
\frac{d P}{d t}=k P \ln \left(\frac{L}{P}\right)
$$

Operacionalizando a equação e realizando a integração, tem-se:

$$
\int \frac{d P}{P \ln \left(\frac{L}{P}\right)}=\int k d t
$$

Introduzindo a mudança de variáveis $u=\ln \left(\frac{L}{P}\right)$ e $-d u=\frac{d P}{P}$, e denominando $c$ como uma constante de integração, tem-se:

$$
\begin{aligned}
& -\int \frac{d u}{u}=\int k d t \\
& -\ln |u|+c=k t
\end{aligned}
$$

Voltando às variáveis originais do problema, obtém-se:

$$
-\ln \left|\ln \left(\frac{L}{P}\right)\right|+c=k t
$$

Como $P_{0}=P(0)$, oferece a seguinte definição da constante de integração:

$$
c=\ln \left|\ln \left(\frac{L}{P_{0}}\right)\right|
$$

A substituição de (25) em (24) e a exploração de manipulações algébricas e da propriedade logarítmica do quociente, implica em:

$$
\begin{gathered}
-\ln \left|\ln \left(\frac{L}{P}\right)\right|+\ln \left|\ln \left(\frac{L}{P_{0}}\right)\right|=k t, \\
\ln \left[\frac{\ln \left(\frac{L}{P_{0}}\right)}{\ln \left(\frac{L}{P}\right)}\right]=k t
\end{gathered}
$$




$$
\ln \left(\frac{L}{P}\right)=e^{-k t} \ln \left(\frac{L}{P_{0}}\right)
$$

Desenvolvendo o processo exponencial em (28), obtém-se:

$$
\begin{gathered}
P=\frac{L}{e^{e^{-k t} \ln \left(\frac{L}{P_{0}}\right)}} \\
P(t)=L e^{e^{-k t} \ln \left(\frac{P_{0}}{L}\right) .}
\end{gathered}
$$

Neste caso, é perceptível uma aproximação dos modelos de Verhulst (logístico) e Gompertz, tendo em vista que $\lim _{t \rightarrow \infty} P(t)=L$ e $\lim _{t \rightarrow 0} P(t)=P_{0}$. Assim:

[...] tanto a curva logística, quanto a curva de Gompertz tendem a capacidade de suporte para tempos grandes. Por apresentar um ponto de inflexão em um valor de população menor do que a curva logística, a curva gerada pelo modelo de Gompertz apresenta um crescimento maior no inicio (sic) do tempo e chega ao equilíbrio mais rapidamente. Dependendo da população estudada, este modelo pode ser o mais adequado (VILLAR, 2014, p. 19).

A comparação gráfica dos três modelos estudados evidencia as principais características de cada uma, como mostra a Figura 1.

Figura 1 - Gráfico comparativo entre as curvas geradas a partir dos modelos de Malthus, Verhulst

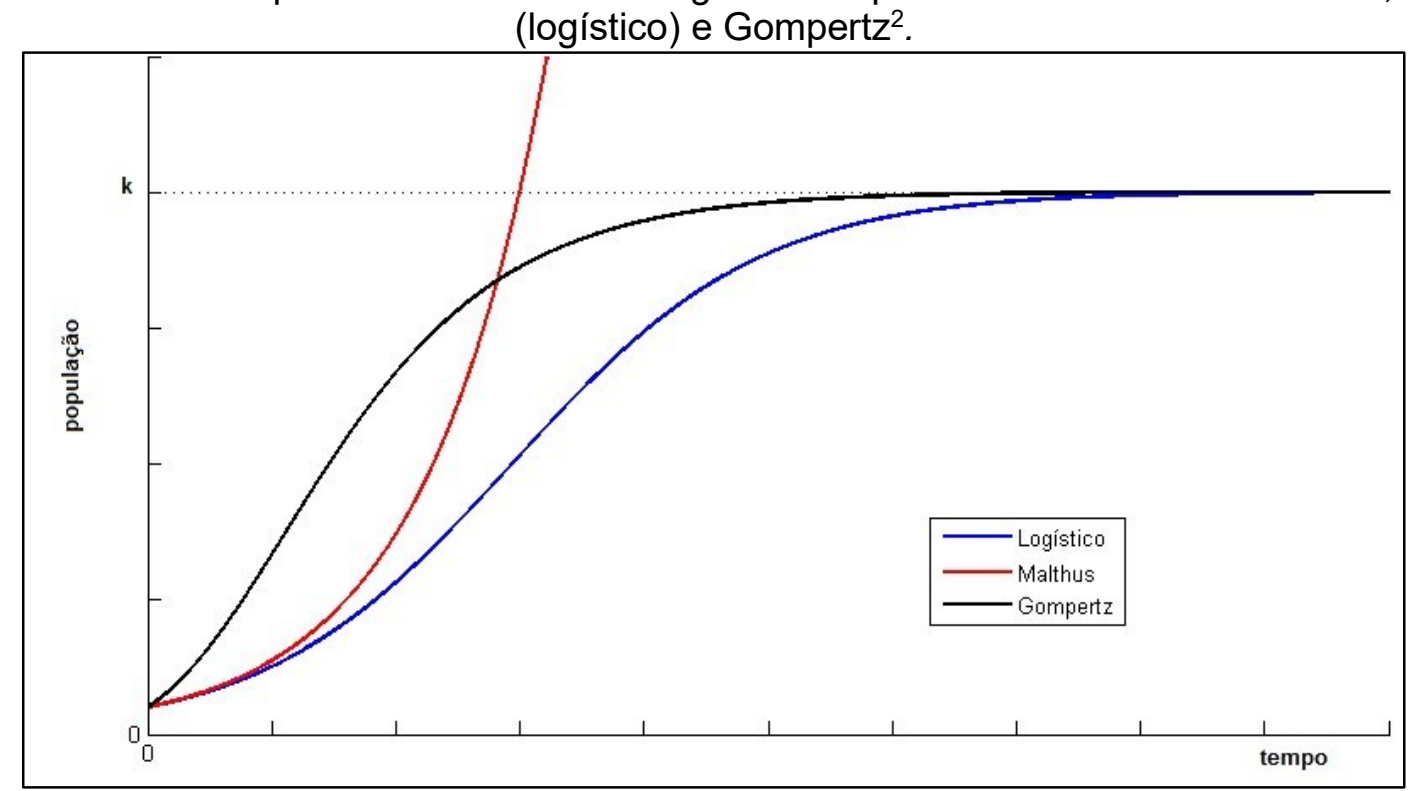

Fonte: VILLAR (2014, p. 26).

Cada um dos modelos apresentados nestas seções será utilizado no presente trabalho a fim de verificar qual o que melhor se adequa às especificidades da população santa-rosense e,

\footnotetext{
2 Villar, em sua dissertação, utiliza $k$ como a representação do limite populacional sustentável, diferente de $L$, adotado neste estudo.
} 
consequentemente, apresenta projeções mais prováveis de serem concretizadas no futuro. Os dados estatísticos utilizados basearam-se em documentos oficiais, principalmente relativos aos relatórios dos censos demográfico realizados pelo Instituto Brasileiro de Geografia e Estatística (IBGE).

\section{Metodologia}

Com vistas aos embasamentos teóricos utilizados para este estudo, desenvolveu-se uma investigação que alia caráter qualitativo e quantitativo. Aquele, justifica-se pela preocupação na identificação e elucidação dos fatores que determinam ou que contribuem para a ocorrência dos fenômenos investigados; este, por ter em vista o estudo e a busca de representações abstratas da dinâmica populacional do município de Santa Rosa, possibilitadas pela exploração de Equações Diferenciais.

Em relação aos procedimentos metodológicos, a pesquisa possui cunho documental e bibliográfico, uma vez que sua fundamentação se dá sobre dados históricos populacionais obtidos junto a órgãos oficiais, além de renomadas obras literárias na área de Modelagem Matemática.

A pesquisa documental trilha os mesmos caminhos da pesquisa bibliográfica, não sendo fácil por vezes distingui-las. A pesquisa bibliográfica utiliza fontes constituídas por material já elaborado, constituído basicamente por livros e artigos científicos localizados em bibliotecas. A pesquisa documental recorre a fontes mais diversificadas e dispersas, sem tratamento analítico, tais como: tabelas estatísticas, jornais, revistas, relatórios, documentos oficiais, cartas, filmes, fotografias, pinturas, tapeçarias, relatórios de empresas, vídeos de programas de televisão, etc. (FONSECA, 2002, p. 32).

Após a revisão bibliográfica e coleta de dados referentes aos censos e estimativas populacionais do referido município, adquiridas junto ao IBGE, se deu a determinação do coeficiente de crescimento populacional relativo à solução analítica das Equações Diferenciais de Malthus, Verhulst e Gompertz. Assim, foi possível a construção e posterior validação de um modelo matemático que relacione a variação populacional em função do tempo, projetando cenários populacionais futuros.

Convém salientar que, para os cálculos realizados, adotou-se como procedimento padrão na representação numérica a utilização de 4 casas decimais. As principais ferramentas utilizadas para as resoluções e representações de cada modelo encontrado foram a Calculadora Científica e os softwares GeoGebra e Maple.

\section{Resultados e discussão}

Ao investigar o crescimento populacional santa-rosense é imprescindível considerar seu contexto histórico de constituição, principalmente relativo à época considerada para o estudo. Geograficamente, o território municipal tem sofrido alterações desde sua emancipação política e econômica, sendo subdividido inicialmente nos distritos de Cruzeiro, Santa Rosa, Sete de Setembro, Campina e Cândido Godói. 
De 1960 até os dias atuais, as principais alterações territoriais municipais foram o desmembramento dos distritos de Campina e Cândido Godói, no ano de 1963, e a posterior junção de Cruzeiro e Santa Rosa - mantendo a nomeação deste último e constituindo a sede municipal (RIO GRANDE DO SUL, 1984). Desta forma, a fim de garantir a eficácia da pesquisa por meio da variação populacional relativa a um mesmo território, a população considerada para o ano de 1960 no estudo foi apenas referente aos distritos que compõem as atuais demarcações geográficas. A Tabela 1 evidencia o crescimento demográfico vivenciado nas últimas cinco décadas e a variação do índice de crescimento a cada década.

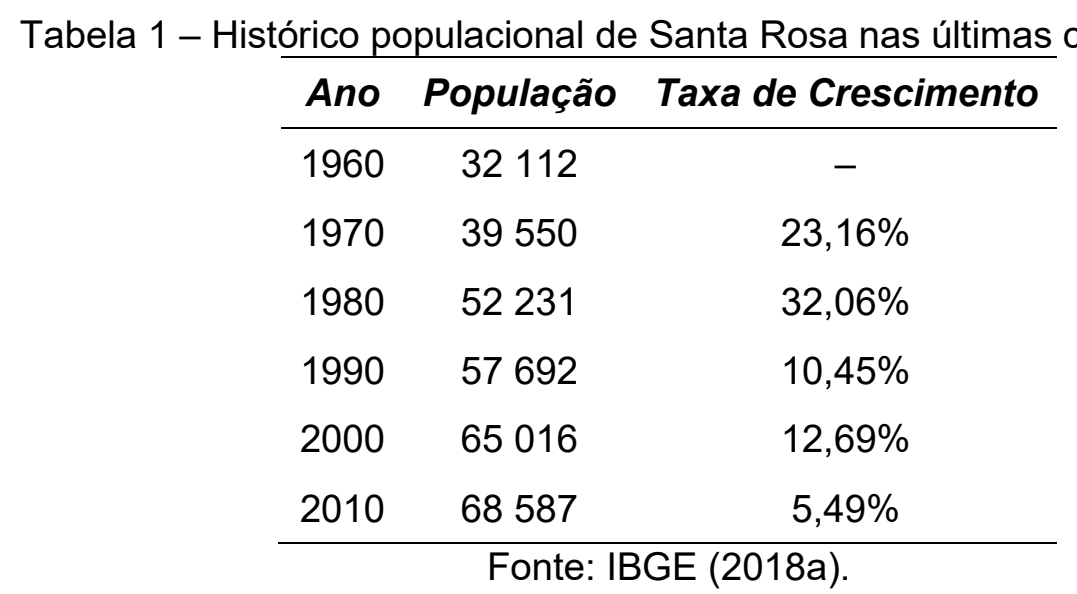

Convém salientar, que no ano de 1990 foi utilizada a estimativa preliminar do IBGE para o período que antecedeu o censo da década, realizado no ano seguinte. Já para a projeção das funções exploradas, as hipótese que nortearam a formulação do modelo matemático foram: a) a taxa de crescimento, $k$, é baseada na primeira década analisada $\left(P_{0}=1960\right.$ e $P_{1}=1970$ ); e b) o limite sustentável da população santa-rosense é de 300000 habitantes.

A primeira hipótese se dá pela necessidade de delimitar um período para definição da variável de crescimento, na qual se usufruiu da conjectura de que a variação da primeira década analisada irá definir tais índices posteriores. Já a segunda, seguiu da análise da queda da taxa de crescimento ao longo das últimas cinco décadas, na qual verificou-se a proximidade do comportamento do referido índice ao longo do tempo, representado na figura a seguir pelos pontos em laranja, com o processo de regressão logarítmica. Utilizando de tal técnica matemática, concluiu-se que demorariam cerca de 10 décadas após 1960 para que a taxa de crescimento se anulasse, passando a ser uma taxa de decrescimento, como mostra a Figura 2. 
Figura 2 - Regressão logarítmica da taxa de crescimento populacional de Santa Rosa em função das décadas a partir de 1960.

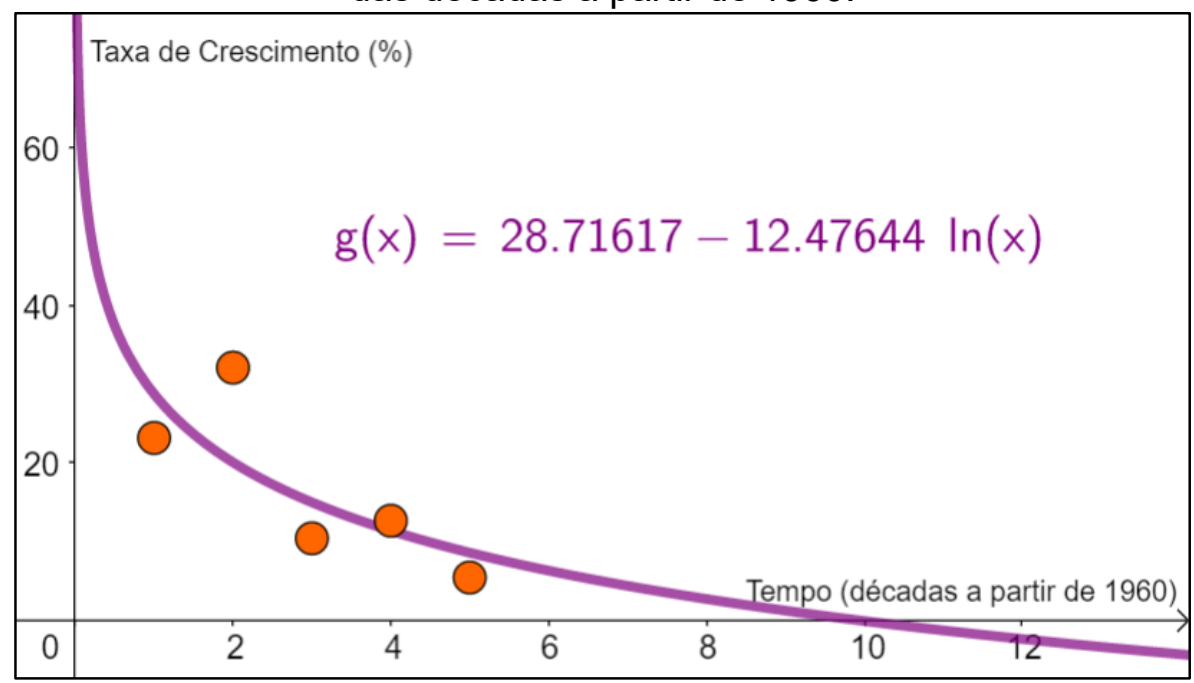

Fonte: Dados da pesquisa.

Seguindo esta análise, o limitante da população considerada seria atingido aproximadamente em $t=10$. Substituindo tal valor no modelo de Malthus, encontramos que a população estaria em quantidade próxima a 300000 - valor utilizado para o parâmetro $L$ nos modelos de Verhulst e Gompertz.

\subsection{Aplicação do modelo de Malthus}

Ao visualizarmos os dados históricos relativos à população santa-rosense e adotarmos o tempo $t$ em décadas a partir de 1960 , teremos $P_{0}=P(0)=32112$ e $P(1)=39550$. Utilizando o modelo de Malthus em sua forma analítica (7), e realizando os devidos procedimentos matemáticos, obtém-se:

$$
\begin{gathered}
P(1)=P_{0} e^{k \cdot 1}, \\
39550=32112 e^{k \cdot 1}, \\
e^{k}=\frac{39550}{32112} \\
k=\ln \frac{39550}{32112} \cong 0,2083 .
\end{gathered}
$$

Com o coeficiente de crescimento $k$ definido, a função malthusiana que retratará a variação populacional em relação ao tempo será:

$$
P(t)=32112 e^{0,2083 k} .
$$




\subsection{Aplicação do modelo de Verhulst}

Ao explorarmos a solução analítica de Verhulst (19), adotando os devidos procedimentos matemáticos, obtém-se:

$$
\begin{gathered}
P(1)=\frac{32112.300000}{(300000-32112) e^{-1 k}+32112}, \\
39550=\frac{32112.300000}{(300000-32112) e^{-k}+32112}, \\
e^{-k}=\frac{(32112.300000)-(39550.32112)}{39550 .(300000-32112)}, \\
k=-\ln \left(\frac{(32112.300000)-(39550.32112)}{39550 .(300000-32112)}\right) \cong 0,2365 .
\end{gathered}
$$

Com o coeficiente de crescimento $k$ definido, a função de Verhulst que retratará a variação populacional em relação ao tempo será:

$$
P(t)=\frac{32112.300000}{(300000-32112) e^{-0,2365 t}+32112}
$$

\subsection{Aplicação do modelo de Gompertz}

Ao explorarmos a solução analítica de Gompertz, adotando as mesmas hipóteses do modelo anterior e usufruindo das operações e procedimentos matemáticos necessários, tem-se:

$$
\begin{gathered}
P(1)=300000 e^{e^{-k \cdot 1} \cdot \ln \left(\frac{32112}{300000}\right),} \\
39550=300000 e^{e^{-k} \cdot \ln \left(\frac{32112}{300000}\right),} \\
e^{e^{-k} \cdot \ln \left(\frac{32112}{300000}\right)}=\frac{39550}{300000}, \\
e^{-k} \cdot \ln \left(\frac{32112}{300000}\right)=\ln \left(\frac{39550}{300000}\right),
\end{gathered}
$$




$$
\begin{gathered}
e^{-k}=\frac{\ln \left(\frac{39550}{300000}\right)}{\ln \left(\frac{32112}{300000}\right)}, \\
k=-\ln \left[\frac{\ln \left(\frac{39550}{300000}\right)}{\ln \left(\frac{32112}{300000}\right)}\right] \cong 0,0979 .
\end{gathered}
$$

Com o coeficiente de crescimento $k$ definido, a função de Gompertz que retratará a variação populacional em relação ao tempo será:

$$
P(t)=300000 e^{e^{-0,0979 t} \cdot \ln \left(\frac{32112}{300000}\right) .}
$$

\subsection{Análise dos modelos matemáticos}

A comparação numérica populacional dos dados históricos relativos ao município santarosense com as projeções desenvolvidas em cada modelo matemático torna-se, neste caso, a principal ferramenta de sua (in)validação, tendo em vista a identificação da margem de erro de cada função e sua respectiva aceitabilidade. Neste sentido, quanto mais próximo dos dados reais, maior a probabilidade de acerto das projeções. A Tabela 2 apresenta tal comparação.

Tabela 2 - Histórico populacional do município de Santa Rosa nas últimas cinco décadas em comparação com os modelos matemáticos encontrados.

\begin{tabular}{cccccccc}
\hline Ano & $\begin{array}{c}\text { População } \\
\text { real }\end{array}$ & $\begin{array}{c}\text { População } \\
\text { calculada via } \\
\text { Malthus }\end{array}$ & $\begin{array}{c}\text { Erro } \\
\text { (\%) }\end{array}$ & $\begin{array}{c}\text { População } \\
\text { calculada via } \\
\text { Verhulst }\end{array}$ & $\begin{array}{c}\text { Erro } \\
\text { (\%) }\end{array}$ & $\begin{array}{c}\text { População calculada } \\
\text { via Gompertz }\end{array}$ & $\begin{array}{c}\text { Erro } \\
\text { (\%) }\end{array}$ \\
\hline 1960 & 32112 & 32112 & 0 & 32112 & 0 & 32112 & 0 \\
1970 & 39550 & 39550 & 0 & 39550 & 0 & 39550 & 0 \\
1980 & 52231 & 48711 & 6,7 & 48400 & 7,3 & 47774 & 8,5 \\
1990 & 57692 & 59994 & 4,0 & 58782 & 1,9 & 56700 & 1,7 \\
2000 & 65016 & 73890 & 13,6 & 70766 & 8,8 & 66228 & 1,9 \\
2010 & 68587 & 91005 & 32,7 & 84339 & 23,0 & 76245 & 11,2 \\
$\Sigma$ & 315188 & 345262 & 9,5 & 333949 & 6,0 & 318609 & 1,1 \\
\hline
\end{tabular}

Fonte: Dados da pesquisa.

A Figura 3 apresenta a comparação gráfica dos dados reais, representados pelos pontos em laranja, com as simulações realizadas, facilitando suas análises e interpretações e ressaltando as curvas definidas por cada modelo explorado. 
Figura 3 - Comparação dos modelos matemáticos utilizados com os dados reais.

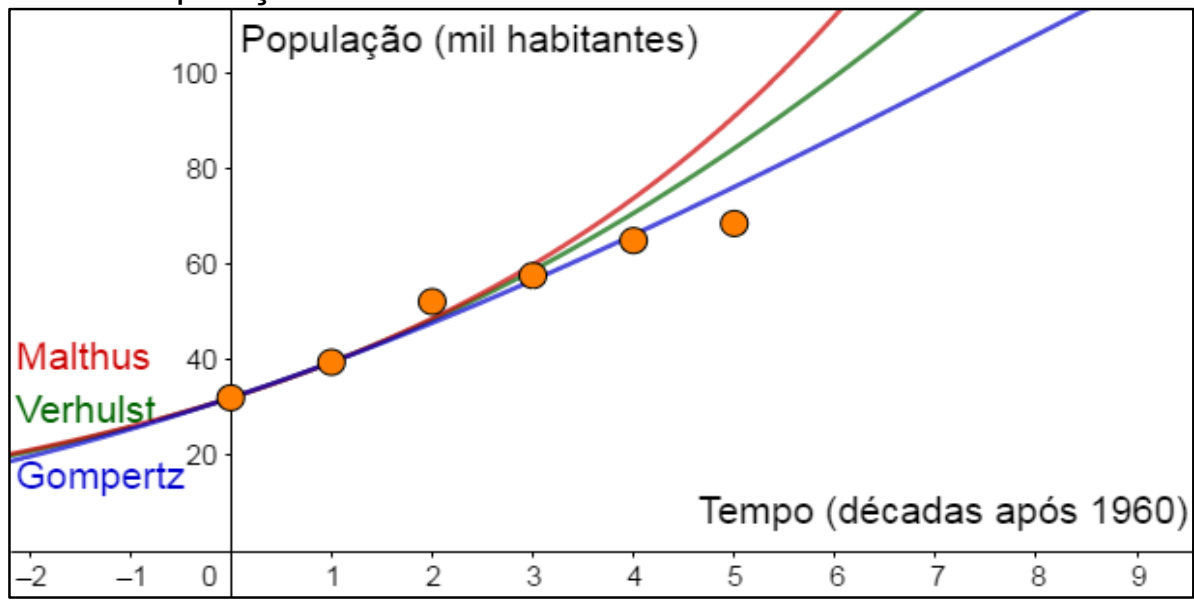

Fonte: Dados da pesquisa.

Ao analisar as representações numéricas e gráficas da utilização dos modelos de Malthus, Verhulst e Gompertz fica nítida uma maior aproximação dos dados históricos com este último, induzindo a crer na existência de um fator de inibição da variável de estado proporcional ao respectivo logaritmo. As projeções populacionais futuras se dão, portanto, a partir da função (47), que descreve a população municipal (habitantes) em função do tempo (décadas a partir de 1960).

Apesar de ter tido um crescimento mais elevado no início de sua colonização e emancipação política, a taxa de variação populacional de Santa Rosa tem decaído nas últimas décadas e encaminhado à estabilização de um determinado limite sustentável. Ressalta-se, assim, a necessidade de investimentos, nos mais diversos setores, que garantam a expansão da cidade, revertendo as tendências de estagnação populacional e impulsionando o progresso municipal e regional.

\section{Considerações finais}

Buscar abstrações que se aproximem da representação de determinado fenômeno tem configurado um grande desafio aos que se aventuram na tarefa de modelar matematicamente. Ao especificar-se às dinâmicas populacionais, a variação dos modos de vida e as diferentes características das populações consideradas tornam-se causas de constantes modificações e aprimoramentos de modelos que sejam válidos e possam ser explorados sob a ótica das mais diferentes áreas de estudo.

Ao analisar o contexto histórico da construção dos modelos de Malthus, Verhulst e Gompertz na dinâmica populacional fica nítida a tentativa de adaptações da realidade por meio de Equações Diferenciais. Tais recursos matemáticos tomam sua relevância na Modelagem Matemática por possibilitarem a representação de relações entre uma variável dependente e pelo menos uma independente - integrando o conceito de função.

Embora, por vezes, exija o ajuste de seus parâmetros, a fim de buscar a minimização da margem de erro em relação às reais proporções do processo considerado, as Equações 
Diferenciais evidenciam suas potencialidades por possibilitarem a adequação a diferentes conjecturas, como por exemplo o incremento de um limite populacional sustentável. Outras técnicas matemáticas, como os diferentes tipos de regressão, muitas vezes, acabam limitando-se ao processo de representação simbólica, sem considerar os princípios que regem os fenômenos considerados e suas respectivas particularidades.

Em relação ao município de Santa Rosa, percebe-se que o comportamento da variação populacional em relação às últimas cinco décadas tem se aproximado à curva de Gompertz, isto é, sido consideravelmente alta no início e baixa no fim do período. Tal fato reforça a necessidade contínua de planejamentos e investimentos nos diferentes setores estruturais da sociedade, a fim de impedir a estagnação populacional do município e garantir o progresso local.

\section{Referências}

BASSANEZI, R. C. Ensino-aprendizagem com Modelagem Matemática. São Paulo: Contexto, 2004.

BIEMBENGUT, M. S.; HEIN, N. Modelagem Matemática no Ensino. São Paulo: Contexto, 2005.

FONSECA, J. J. S. Metodologia da pesquisa científica. Fortaleza: UEC, 2002.

IBGE. Censos Demográficos. Disponível em:

<https://www.ibge.gov.br/estatisticas/sociais/populacao/>. Acesso em: 25 nov. 2018.

IBGE. Projeções da população: Brasil e unidades da federação. Revisão 2018. 2. ed. Rio de Janeiro: IBGE, 2018.

RIO GRANDE DO SUL. Secretaria de Coordenação e Planejamento. De Província de São Pedro a Estado do Rio Grande do Sul: Censos do RS: 1960-1980. Porto Alegre: Fundação de Economia e Estatística, 1984.

SANTA ROSA. Município: História. Disponível em <http://www.santarosa.rs.gov.br/municipio.php>. Acesso em: 15 nov. 2018.

VILLAR, R. P. Estudo Analítico da Equação de Fischer Linearizada: Determinação de Tamanhos Mínimos de Fragmentos Populacionais. 2014. 73 f. Dissertação (Mestrado em Ciências e Engenharia Ambiental) - Universidade Federal de Alfenas, Poço de Caldas, 2014. 